\title{
First Two-Loop Amplitudes with the Numerical Unitarity Method
}

\author{
Samuel Abreu ${ }^{a}$, Fernando Febres Cordero ${ }^{a}$, Harald Ita ${ }^{a}$, Matthieu Jaquier ${ }^{a}$, Ben \\ Page $^{*, a}$, Mao Zeng ${ }^{b}$ \\ ${ }^{a}$ Physikalisches Institut, Albert-Ludwigs Universität Freiburg, D-79104 Freiburg, Germany \\ ${ }^{b}$ Mani L. Bhaumik Institute for Theoretical Physics, UCLA Department of Physics and \\ Astronomy, Los Angeles, CA 90095, USA \\ E-mail: ben.page@physik.uni-freiburg.de
}

\begin{abstract}
The numerical unitarity approach, which allows for the complete reduction of phenomenologically-relevant scattering amplitudes to linear combinations of master integrals, has recently been extended to two loops. In these proceedings we discuss the method and a proof-of-principle calculation of the four-gluon amplitudes in an arbitrary helicity configuration. Furthermore, we combine the method with functional reconstruction techniques to extract analytic formulae for the amplitudes.
\end{abstract}

13th International Symposium on Radiative Corrections (Applications of Quantum Field Theory to Phenomenology)

25-29 September, 2017

St. Gilgen, Austria

${ }^{*}$ Speaker. 


\section{Introduction}

In the coming years, the scientific program of the LHC is projected to produce measurements of unprecedented precision. On the theoretical side, in order to match the percent level uncertainty demands of the experiments, an increasing number of theoretical predictions in QCD will need to be calculated at NNLO accuracy.

Thanks to new advances in the understanding of how to handle real-radiation contributions, there have been a number of new 2-to-2 NNLO computations in recent years. Nevertheless, there are still open questions on how to move beyond the current state of the art such as allowing access to kinematic dependence through recoiling jets or internal mass effects. At the level of the virtual contributions this implies the computation of increasingly complex multi-scale two-loop scattering amplitudes.

In phenomenologically-relevant two-loop calculations, the technologies employed for the evaluation of scattering amplitudes are often based on Feynman diagram techniques. The procedure starts by generating an analytic integrand and applying a series of tensor reduction [1] and integration-by-parts [2] (IBP) identities in order to express the amplitude as a linear combination of master integrals. Once this reduction is achieved, it remains to integrate the master integrals themselves, which can be achieved using, for example, the differential equations method $[3,4,5,6]$. This procedure is very general, but involves generating large analytic expressions for the integrands, to which one applies complicated identities. Further, the generation of IBP identities, usually achieved with the Laporta algorithm [7], is often computationally demanding and unenlightening. This situation is reminiscent of the landscape of phenomenologically-relevant one-loop amplitude calculations before the so-called "NLO revolution" of the last decade.

The numerical unitarity method aims to remedy these difficulties at two loops, building on its successes at one loop. The approach performs reduction and evaluation simultaneously, providing direct access to (physical) master integral coefficients. Further, the numerical nature of the method means that it is less sensitive to increasing numbers of scales in the problem. In these proceedings we present the method and its recent successful application in a proof of principle numerical computation of the leading-colour contributions to four-gluon helicity amplitudes at two loops [10].

\section{The Numerical Unitarity Method}

The numerical unitarity method is an approach to calculating scattering amplitudes. It achieves a full reduction to master integrals at the integrand level, building the amplitude from physical objects - products of trees. The principal idea is to start with a special ansatz for the integrand of a gauge theory amplitude and to constrain it through knowledge of the on-shell limits. In full generality the rational ansatz reads:

$$
\mathscr{A}\left(\ell_{l}\right)=\sum_{\Gamma} \sum_{i \in M_{\Gamma} \cup S_{\Gamma}} \frac{c_{\Gamma, i} m_{\Gamma, i}\left(\ell_{l}\right)}{\prod_{\text {props } j} \rho_{j}} .
$$

That is, we write the integrand of the amplitude as a linear combination of rational functions whose denominators are given by all possible propagator structures $\Gamma$. Furthermore, in order to facilitate an automatic reduction to master integrals, we decompose the numerator into a collection of 'master 
integrands' $\left(M_{\Gamma}\right)$ and 'surface terms' $\left(S_{\Gamma}\right)$, which integrate to zero. The ansatz coefficients are then numerically fixed by comparison against on-shell data. When taking a set of propagators on-shell, $\ell_{l} \rightarrow \ell_{l}^{\Gamma}$, the leading contribution to the integrand in this limit factorises into a product of tree amplitudes,

$$
\sum_{\text {states }} \prod_{i \in T_{\Gamma}} \mathscr{A}_{i}^{\text {tree }}\left(\ell_{l}^{\Gamma}\right)=\sum_{\substack{\text { ancestors } \\ i \in M_{\Gamma^{\prime}} \cup \Gamma_{\Gamma^{\prime}},}} \frac{c_{\Gamma^{\prime}, i} m_{\Gamma^{\prime}, i}\left(\ell_{l}^{\Gamma}\right)}{\prod_{\text {props } j} \rho_{j}} .
$$

With such a tool, one can numerically generate a constraining set of linear equations for the coefficients of master integrals, which allows us to extract the physical information directly, avoiding intermediate complexity. The integration of the amplitude can now be achieved by inserting any available set of master integrals. Many of the facets of this procedure are familiar from the one-loop incarnation, but there are new challenges to solve at two loops due to more complicated integral relations and difficult to access ansatz coefficients.

\subsection{Fixing the Integrand From On-Shell Data}

The factorisation properties of the loop integrand allow one to hierarchically construct many numerator functions. By first taking a factorisation limit involving a maximal number of propagators, equation (2.2) involves only one numerator function. When moving down the hierarchy level by level, it is then almost always the case that higher level contributions are known beforehand. However, the one-to-one correspondence between products of trees and integrand contributions only holds for leading poles in the factorisation limit and beyond one-loop this procedure must be modified. Indeed, at two loops and beyond, certain factorisation limits also have sub-leading contributions, which are not constrained in the limit.

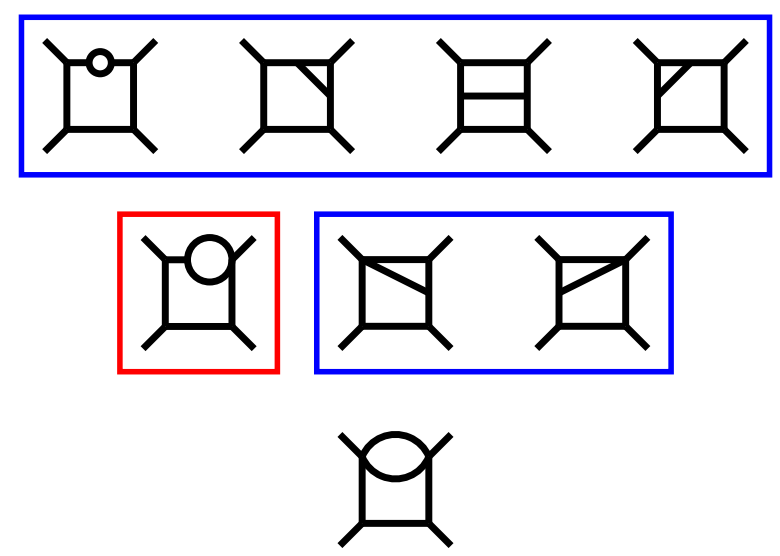

Figure 1: A subset of the full hierarchy of four-gluon amplitude propagator structures. The numerator associated to the topology in red is extracted from the descendent cut as described in the text.

In order to solve this problem, we extend the standard ansatz fitting procedure. Consider the sub-hierarchy given in figure 1 . All propagator structures in blue can be accessed using the standard subtraction procedure. However, the propagator structure in red is not associated to a product of trees because it is a 'sub-leading pole' contribution, sharing the same set of propagators with the corresponding leading pole in the top left of figure 1, but raised to different powers. In order to 
extract this contribution we enlarge the ansatz for the cut equation of the descendent topology at the bottom of figure 1 to simultaneously extract its numerator and that of the sub-leading contribution. Further details can be found in [11].

\subsection{Master/Surface Decomposition}

The remaining ingredient in extending numerical unitarity to two loops is the construction of a complete master/surface decomposition of the integrand, see equation (2.1). To achieve this, we need to be able to construct the full set of surface terms corresponding to a given set of propagators. General surface terms are IBP relations. These follow from the fact that total derivatives integrate to zero in dimensional regularisation,

$$
0=\int \prod_{l=1,2} d^{D} \ell_{l} \frac{\partial}{\partial \ell_{j}^{v}}\left[\frac{u_{j}^{v}}{\prod_{\text {props } k} \rho_{k}}\right] .
$$

Here we call $u_{j}^{v}$ an 'IBP vector'. Unfortunately, for an arbitrary IBP vector the resulting relations correspond to multiple propagator structures, due to the raised propagator powers arising from the chain rule when the derivative acts on the propagator. To avoid this, we construct a restricted set of IBP relations, by employing the strategy of [12]. The key idea is to search for IBP-vectors $u_{j}^{v}$ which do not raise the propagator power as they satisfy the 'non-doubling relation',

$$
u_{i}^{v} \frac{\partial}{\partial \ell_{i}^{v}} \rho_{j}=f_{j} \rho_{j},
$$

where there is no summation over $j$. Such a vector is deceptively hard to find as it must be polynomially valued in order to produce numerator structures in equation (2.3). The principal difficulty in solving equation (2.4) is that we are not allowed to divide by polynomials in loop momenta and so this is not a problem of linear algebra, but algebraic geometry. We solve these equations by moving to 'adapted coordinates' [13]. This is an overcomplete coordinate system of inverse propagators and irreducible scalar products,

$$
\ell_{l}^{\mu} \rightarrow\left(\rho_{i}, \alpha_{j}, \mu_{n m}\right)
$$

Notably this transformation involves increasing the number of coordinates beyond the number of degrees of freedom. This redundancy is removed by working modulo the (polynomial) constraint equations,

$$
\mu_{l l}=\left(\mu_{l}\right)^{2}=\rho_{l 0}-\sum_{v=0}^{3} \ell_{l}^{v} \ell_{l v} .
$$

In these coordinates it is simple to satisfy equation (2.4) by writing IBP vectors as:

$$
u_{i}^{v} \frac{\partial}{\partial \ell_{i}^{v}}=f_{i} \rho_{i} \frac{\partial}{\partial \rho_{i}}+u_{j} \frac{\partial}{\partial \alpha_{j}}+\sum_{l, l^{\prime}=1,2} f_{l^{\prime}}^{l} \vec{\mu}_{l} \cdot \frac{\partial}{\partial \vec{\mu}_{l^{\prime}}} .
$$

Due to the constrained nature of the coordinate system the vectors also satisfy compatibility conditions

$$
0=f_{i} \rho_{i} \frac{\partial \mu_{n m}}{\partial \rho_{i}}+u_{j} \frac{\partial \mu_{n m}}{\partial \alpha_{j}}-\sum_{l, l^{\prime}=1,2} f_{l^{\prime}}^{l} \vec{\mu}_{l} \cdot \frac{\partial \mu_{n m}}{\partial \vec{\mu}_{l^{\prime}}} .
$$


The set of solutions to these equations form a so-called 'module', for which we can find a generating set using a package such as SINGULAR [14]. We can then use these vectors to build arbitrary surface terms, by inserting linear combinations of the generators with polynomial coefficients into the total derivative. Related techniques can be found in [15].

\subsection{Regulator Dependence of Coefficients}

The coefficients of master integrals in the decomposition of equation (2.1) also depend on the dimensional regulators. In the numerical unitarity approach, this dependence can be understood as arising from two different sources - the on-shell data used to fix the ansatz, and the surface terms themselves.

The first source is familiar from one loop - the integrand will generically depend on the dimension of the internal polarisation states. At two loops the integrand is at most quadratic in $D_{s}$. The functional dependence on this parameter can be reconstructed through a simple two-loop generalisation of the procedure of [16], where one reconstructs the quadratic polynomial by sampling over three different values of $D_{s}$. In practice $D_{s}$ must be greater than or equal to the embedding dimension of the loop momenta, which at two loop is at least 6 , and so the values we use are $D_{s}=6,7,8$. With analytic control over this parameter we then set $D_{s}=D$, recovering the 't Hooft-Veltman scheme of dimensional regularisation.

The second source of $D$-dependence arises because the surface terms have an explicit dependence on $D$. This is then inherited by the coefficients, which are rational functions of $D$. Notably, this set of univariate rational functions turns out to only be kinematically dependent in the numerator, i.e. all coefficients take the form

$$
c(D)=\frac{P\left(D, s_{i j}\right)}{Q(D)}=\frac{p_{0}\left(s_{i j}\right)+p_{1}\left(s_{i j}\right) D+\cdots+p_{i}\left(s_{i j}\right) D^{i}}{q_{0}+q_{1} D+\cdots+q_{j-1} D^{j-1}+D^{j}} .
$$

The problem of how to reconstruct a rational functions has recently received attention in the literature $[17,18]$. The main observation is that the values of the coefficients can be inferred from the value that the function takes at a finite number of points. Therefore, by evaluating the coefficient at a set of (in principle arbitrary) distinct points $D_{i}$ one reconstructs the full function. In practice we use the formula of Thiele [17, 19], expressing $c(D)$ in the form of a continued fraction,

$$
c(D)=a_{0}+\frac{D-D_{0}}{a_{1}+\frac{D-D_{1}}{a_{2}+\frac{D-D_{2}}{\cdots+\frac{D-D_{N-1}}{a_{N}}}}} .
$$

Simple relations then exist between the coefficients $a_{i}$ and a set of evaluations of the coefficient function $c\left(D_{i}\right)$. Importantly, we fix the kinematically independent denominator from a single value of the $s_{i j}$ and henceforth only polynomial reconstruction techniques are necessary.

\section{Proof of Principle - Two-Loop Four-Gluon Scattering Amplitudes}

In order to show that the numerical unitarity approach is viable in phenomenologically-relevant calculations, we have applied the method to numerically recompute the planar two-loop four-gluon 
amplitudes in the leading-colour approximation with an arbitrary helicity configuration. These were first computed in [20,21], and exhibit the full complexity of massless phenomenologicallyrelevant amplitudes, providing an excellent example to test the approach.

\subsection{Implementation}

Our approach is implemented in a $\mathrm{C}++$ code in order to allow for the necessary rapid numerical evaluation. The products of tree amplitudes are calculated through the use of a Berends-Giele recursion [22], which allows for good computational complexity when appropriately cached. Furthermore, this allows for flexibility in the particle content as well as for simple varying of the value of $D_{s}$ in order to implement the associated polynomial reconstruction. The colour decomposition is performed using the approach of [23], which is then expanded to the leading order in $N_{c}$.

The hierarchical procedure described in section 2.1 results in a linear function system for coefficients of master integrals and surface terms in each topology [11]

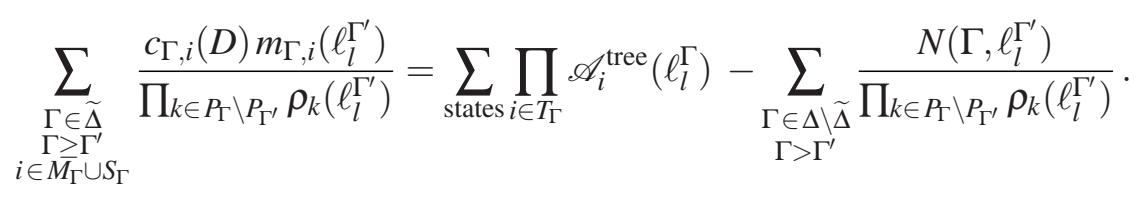

For fixed values of $D, D_{s}$ and the external kinematics, we sample the system over randomly generated on-shell phase-space points in order to constrain the coefficients $c_{\Gamma, i}(D)$. For $n$ unknown coefficients it is enough to sample $n$ points and solve the linear system using PLU factorisation. However, it is sometimes useful to overdetermine the system by sampling $m>n$ points, as this can often lead to a more stable coefficient determination when employing QR factorisation to solve the system numerically. We employ the implementations in LAPACK [24] and MPACK [25] for double and double-double/quad-double precision respectively. A single solution of the linear system evaluates the coefficients for fixed values of the dimensional regulator. These are then used as input for the techniques of section 2.3, which provide the full $D$-dependent physical coefficient.

\subsection{Results and Stability}

The described procedure determines the amplitude for a given numerical value of the kinematics, expressed as a linear combination of master integrals. The functional dependence of the coefficients on the dimensional regulator is exact. At 2-to-2 the resulting form is:

$$
\begin{aligned}
& A(D)=c_{0}(\exists) I_{0}(\exists)+c_{1}(\exists) I_{1}(\exists)+c(\boldsymbol{\beta}) I(\boldsymbol{Q})+c(\times) I(\times) \\
& +c(\nabla) I(\nabla)+c(\infty \propto) I(\infty \circ)+c(\varnothing) I(\diamond)+(s \leftrightarrow t) .
\end{aligned}
$$

For arbitrary kinematic points we have cross checked that the coefficients numerically agree with the values in the literature. We then substitute the known master integrals and perform the Laurent expansion in $\varepsilon=(4-D) / 2$. As an example, for the kinematic point $s=-\frac{3}{4}$ and $t=-\frac{1}{4}$, setting the strong coupling $g$ and renormalization scale $\mu$ to $g=\mu=1$, we find the results in table 1 . These reproduce the prediction of the infra-red pole structure from the universal formula of [26].

We tested the stability of the method over phase space by generating 10000 phase space points using the RAMBO algorithm [27], comparing the result to the available analytic expressions. We 


\begin{tabular}{|c||c|c|c|c|c|}
\hline $\mathscr{A} /\left(\mathscr{A}_{0} N_{c}^{2}\right)(4 \pi)^{4}$ & $\varepsilon^{-4}$ & $\varepsilon^{-3}$ & $\varepsilon^{-2}$ & $\varepsilon^{-1}$ & $\varepsilon^{0}$ \\
\hline \hline$\left(1_{g}^{-}, 2_{g}^{+}, 3_{g}^{-}, 4_{g}^{+}\right)$ & 8.00000 & 55.6527 & 176.009 & 332.296 & 486.502 \\
\hline$\left(1_{g}^{-}, 2_{g}^{-}, 3_{g}^{+}, 4_{g}^{+}\right)$ & 8.00000 & 55.6527 & 164.642 & 222.327 & -8.39044 \\
\hline
\end{tabular}

Table 1: Values for four-gluon two-loop amplitudes evaluated at $s=-\frac{3}{4}$ and $t=-\frac{1}{4}$, setting $g=\mu=1$.

implemented a rescue system using higher precision arithmetics when the $1 / \varepsilon$ pole is precise to less than 2 digits. In figure 2 we show the stability for the least stable helicity configuration, --++ . We see that the stability of the result decreases for higher orders in the $\varepsilon$ series. For the finite part, the distribution is peaked at slightly above 4 digits accuracy.
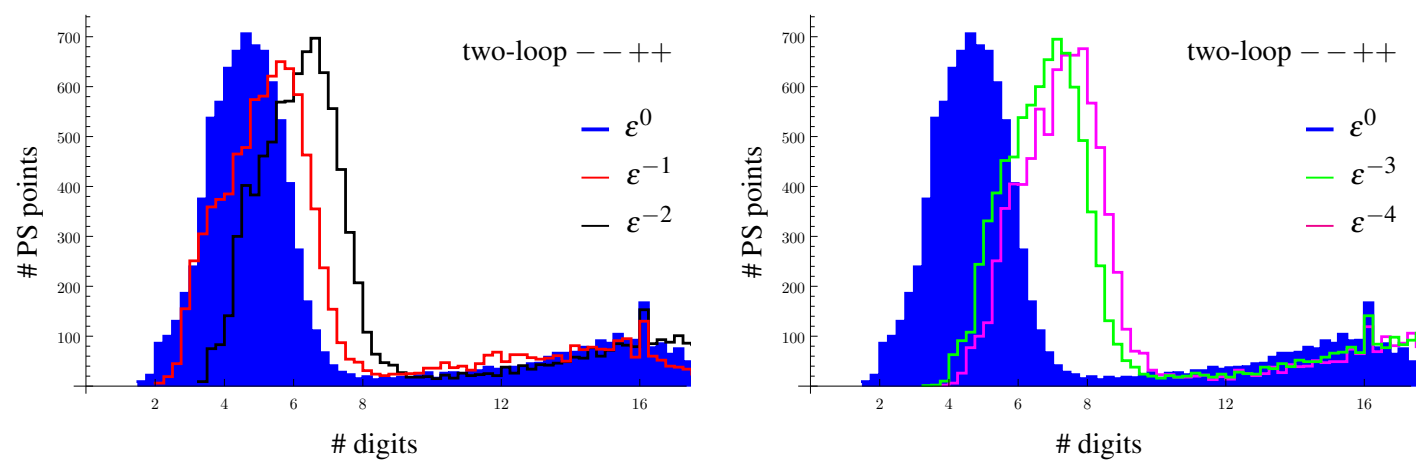

Figure 2: Plot of the stability of the numerical calculation for the --++ helicity configuration.

\section{Analytic Reconstruction}

The integral coefficients themselves are rational functions of the kinematics. In the particular case of a two-scale process and after scaling out the mass dimension, the kinematic dependent coefficients in equation (3.2) are univariate rational functions of the ratio $x=\frac{t}{s}$. As such, these functions are amenable to the univariate functional reconstruction techniques described in section 2.3. Similar approaches have been applied to the reconstruction from numerical samples of the full integrand [17] as well as IBP relations [18]. We applied this technique to reconstruct the full set of master integral coefficients, extracting analytic results from our numerical approach. In practice this required only 15 evaluations of the amplitude, using quad double-precision arithmetic. As an example we present the coefficient of the scalar double box normalised to the mass dimension,

$$
c_{0}(\exists)=\frac{9 x+\frac{\varepsilon\left(-x^{3}-\frac{32 x^{2}}{11}-\frac{97 x}{44}-\frac{5}{22}\right)}{\frac{x^{2}}{33}+\frac{2 x}{33}+\frac{1}{33}}+\frac{\varepsilon^{2}\left(-x^{3}-\frac{385 x^{2}}{51}-\frac{937 x}{102}-\frac{77}{34}\right)}{-\frac{2 x^{2}}{51}-\frac{4 x}{51}-\frac{2}{51}}+\cdots}{-9+66 \varepsilon-184 \varepsilon^{2}+240 \varepsilon^{3}-144 \varepsilon^{4}+32 \varepsilon^{5}}
$$

The full collection of amplitude coefficients for the presented helicity configurations are provided in the ancillary files of [10], which we have checked reproduce the results of [21]. 


\section{Conclusions}

In these proceedings we have described the recent extension of the numerical unitarity method to two loops as well as a proof-of-principle application to the four-gluon scattering amplitudes. The approach is designed to allow a numerical reduction to master integrals for phenomenologicallyrelevant two-loop amplitudes. The method constructs a complete set of surface terms for each integral topology using techniques from algebraic geometry. Furthermore, we have shown the practical possibility of reconstructing analytic formulae for a full scattering amplitude from a numerical approach. The method has since been used to calculate the two-loop five-gluon scattering amplitudes [28] and in the future we hope to apply the method to the calculation of other, as yet uncalculated, phenomenologically-relevant scattering amplitudes.

\section{Acknowledgments}

The work of S.A., F.F.C., M.J. and B.P. is supported by the Alexander von Humboldt Foundation, in the framework of the Sofja Kovalevskaja Award 2014, endowed by the German Federal Ministry of Education and Research. The work of H.I. is supported by a Marie Skłodowska-Curie Action Career-Integration Grant PCIG12-GA-2012-334228 of the European Union. The work of M.Z. is supported by the U.S. Department of Energy under Award Number DE-SC0009937.

\section{References}

[1] G. Passarino and M. J. G. Veltman, Nucl. Phys. B 160 (1979) 151. doi:10.1016/0550-3213(79)90234-7

[2] K. G. Chetyrkin and F. V. Tkachov, Nucl. Phys. B 192 (1981) 159. doi:10.1016/0550-3213(81)90199-1

[3] A. V. Kotikov, Phys. Lett. B 254 (1991) 158. doi:10.1016/0370-2693(91)90413-K

[4] E. Remiddi, Nuovo Cim. A 110 (1997) 1435 [hep-th/9711188].

[5] T. Gehrmann and E. Remiddi, "Differential equations for two loop four point functions," Nucl. Phys. B 580 (2000) 485 [hep-ph/9912329];

[6] J. M. Henn, Phys. Rev. Lett. 110 (2013) 251601 doi:10.1103/PhysRevLett.110.251601 [arXiv:1304.1806 [hep-th]].

[7] S. Laporta, Int. J. Mod. Phys. A 15 (2000) 5087 doi:10.1016/S0217-751X(00)00215-7, 10.1142/S0217751X00002157 [hep-ph/0102033].

[8] G. Ossola, C. G. Papadopoulos and R. Pittau, Nucl. Phys. B 763 (2007) 147 doi:10.1016/j.nuclphysb.2006.11.012 [hep-ph/0609007].

[9] C. F. Berger, Z. Bern, L. J. Dixon, F. Febres Cordero, D. Forde, H. Ita, D. A. Kosower and D. Maître, "An Automated Implementation of On-Shell Methods for One-Loop Amplitudes," Phys. Rev. D 78 (2008) 036003 [arXiv:0803.4180 [hep-ph]].

[10] S. Abreu, F. Febres Cordero, H. Ita, M. Jaquier, B. Page and M. Zeng, Phys. Rev. Lett. 119 (2017) no.14, 142001 doi:10.1103/PhysRevLett.119.142001 [arXiv:1703.05273 [hep-ph]]. 
[11] S. Abreu, F. Febres Cordero, H. Ita, M. Jaquier and B. Page, "Subleading Poles in the Numerical Unitarity Method at Two Loops," arXiv:1703.05255 [hep-ph].

[12] J. Gluza, K. Kajda and D. A. Kosower, “Towards a Basis for Planar Two-Loop Integrals,” Phys. Rev. D 83 (2011) 045012, [arXiv:1009.0472 [hep-th]].

[13] H. Ita, “Two-loop Integrand Decomposition into Master Integrals and Surface Terms,” Phys. Rev. D 94, no. 11, 116015 (2016) [arXiv:1510.05626 [hep-th]].

[14] W. Decker, G.-M. Greuel, G. Pfister, H. Schönemann. 2015. "Singular 4-0-2. A computer algebra system for polynomial computations.” http://www.singular.uni-kl.de

[15] K. J. Larsen and Y. Zhang, "Integration-by-parts reductions from unitarity cuts and algebraic geometry,” Phys. Rev. D 93 (2016) no.4, 041701 [arXiv:1511.01071 [hep-th]].

[16] W. T. Giele, Z. Kunszt and K. Melnikov, "Full one-loop amplitudes from tree amplitudes,” JHEP 0804 (2008) 049 [arXiv:0801.2237 [hep-ph]].

[17] T. Peraro, "Scattering amplitudes over finite fields and multivariate functional reconstruction," JHEP 1612, 030 (2016) [arXiv:1608.01902 [hep-ph]].

[18] A. von Manteuffel and R. M. Schabinger, Phys. Lett. B 744 (2015) 101 doi:10.1016/j.physletb.2015.03.029 [arXiv:1406.4513 [hep-ph]].

[19] M. Abramowitz and I. A. Stegun, "Handbook of mathematical functions: with formulas, graphs, and mathematical tables," 55, 1964, Courier Corporation.

[20] E. W. N. Glover, C. Oleari and M. E. Tejeda-Yeomans, "Two loop QCD corrections to gluon-gluon scattering,” Nucl. Phys. B 605 (2001) 467 [hep-ph/0102201].

[21] Z. Bern, A. De Freitas and L. J. Dixon, “Two loop helicity amplitudes for gluon-gluon scattering in QCD and supersymmetric Yang-Mills theory,” JHEP 0203 (2002) 018 [hep-ph/0201161].

[22] F. A. Berends and W. T. Giele, "Recursive Calculations for Processes with n Gluons," Nucl. Phys. B 306 (1988) 759.

[23] A. Ochirov and B. Page, "Full Colour for Loop Amplitudes in Yang-Mills Theory," JHEP 1702, 100 (2017) [arXiv:1612.04366 [hep-ph]].

[24] E. Anderson, Z. Bai, C. Bischof, S. Blackford, J. Demmel, J. Dongarra, J. Du Croz, A. Greenbaum, S. Hammarling, A. McKenney and D. Sorensen, "LAPACK Users' Guide," Society for Industrial and Applied Mathematics, 1999, ISBN 0-89871-447-8.

[25] M. Nakata, "The MPACK (MBLAS/MLAPACK); a multiple precision arithmetic version of BLAS and LAPACK," http://mplapack.sourceforge.net/.

[26] S. Catani, “The Singular behavior of QCD amplitudes at two loop order," Phys. Lett. B 427 (1998) 161 [hep-ph/9802439].

[27] R. Kleiss, W. J. Stirling and S. D. Ellis, Comput. Phys. Commun. 40 (1986) 359. doi:10.1016/0010-4655(86)90119-0

[28] S. Abreu, F. Febres Cordero, H. Ita, B. Page and M. Zeng, arXiv:1712.03946 [hep-ph]. 